\title{
Was ist Religion? Eine kritische Diskussion
}

Detlef Pollack

Der Artikel diskutiert oft verhandelte Probleme der Erstellung eines wissenschaftlich brauchbaren Religionsbegriffs und unterbreitet einen Vorschlag zur Lösung einiger dieser Probleme. In Abgrenzung von Positionen, die von der Entbehrlichkeit eines allgemeinen Religionsbegriffes ausgehen, trägt er zunächst Argumente zusammen, die für die Erarbeitung einer wissenschaftlich verwendbaren Religionsdefinition sprechen. Dann diskutiert er in Religionswissenschaft und Religionssoziologie gebräuchliche Ansätze zur Definition von Religion: substanzielle, funktionalistische und diskursive Ansätze. Schließlich entwirft er einen eigenen Vorschlag zur Religionsdefinition, der substanzielle und funktionalistische Argumente kombiniert und den Anspruch erhebt, definitorische Trennschärfe und Weite miteinander zu verbinden und sowohl an das Selbstverständnis der Religionsangehörigen anzuknüpfen als es auch zu überschreiten.

Schlagworte: Religionsdefinition, Funktionale Analyse, Substantielle Analyse, Diskurstheorie

\section{Allgemeine Überlegungen zur Definierbarkeit von Religion: Probleme der Religionsdefinition}

Eine universell gültige, allgemein anerkannte Religionsdefinition wird man in den Religionswissenschaften und den mit Religion befassten Sozialwissenschaften vergeblich suchen. ${ }^{1}$ Die Zahl der unterbreiteten Definitionsvorschläge geht in die Hunderte, und es hat sich in den religionswissenschaftlichen Handbüchern und Einführungswerken zu einem gern gepflegten quasi-religiösen Ritual entwickelt, auf die von James Leuba (1921) vor bereits fast 100 Jahren zusammengestellte Sammlung von 48 Religionsdefinitio-

\footnotetext{
${ }^{1}$ So auch Hartmut Zinser (2010: 35): „Der Begriff der Religion ist völlig umstritten. Die Religionswissenschaft hat sich bis heute nicht auf eine Definition verständigen können.“
} 
nen zu verweisen, der man angesichts ihrer inzwischen weiter vorangeschrittenen Vermehrung nicht noch einen Vorschlag hinzufügen möchte. Manch einen hält die Vielfalt der Definitionsversuche nicht davon ab, zumindest einen Arbeitsbegriff von Religion zu entwerfen (Figl 2003: 77). Andere verzichten auf eine eindeutige Definition von Religion und begnügen sich damit, ein „offenes Konzept“, eine Art Raster oder Rahmen für die Bestimmung des Religionsbegriffs zugrunde zu legen und Grundprobleme im Umgang mit dem Religionsbegriff zu diskutieren ${ }^{2}$. Nicht wenige allerdings halten den Versuch, Religion zu definieren, für grundsätzlich zum Scheitern verurteilt, und geben ihn daher auf ${ }^{3}$.

Von den ausgemachten Problemen bei der Bestimmung eines allgemeingültigen Religionsbegriffs seien drei hier herausgestellt. Zum ersten wird immer wieder geltend gemacht, dass sich die Mannigfaltigkeit religiöser Rituale, Symbole, Dogmen, Erfahrungen, Institutionen, Gemeinschaften und Rollen nicht einem einheitlichen Begriff fügen würde und daher die Erstellung eines allgemeingültigen Religionsbegriffs notwendigerweise an der analytischen Uneinfangbarkeit des empirischen Materials scheitern müsse. Schon aufgrund der Vielfalt religiöser Formen und Inhalte sei es ausgeschlossen, sich auf eine einheitliche Religionsdefinition zu verständigen. Die Pluralität möglicher Religionsbegriffe sei prinzipiell unabschließbar (Wagner 1986). Die Bestimmung eines umfassenden Begriffs von Religion sei zweitens aber auch dadurch erschwert, dass es Religionen nur im Plural, nicht in der Einzahl, gebe (Kaufmann 1989: 77). Von der Religion zu sprechen, bedeute, Religion als ein Abstraktum zu behandeln, das es so in der historischen und sozialen Wirklichkeit nicht gebe. In der Zeit der Aufklärung habe die Philosophie zwar versucht, ein hinter den Erscheinungen liegendes allgemeines ,Wesen der Religion" ausfindig zu machen, von dem her sie die historischen Besonderheiten und Absonderlichkeiten kritisieren konnte. Ein solches „Wesen der Religion“, obschon zum Maßstab der Beurteilung geschichtlicher Gestaltungen des Religiösen verwendet, komme in

\footnotetext{
${ }^{2}$ Vgl. Waardenburg 1993: 33; Hock 2011: 10, 20.

${ }^{3}$ Vgl. Kippenberg 1983: 10ff.; Arnal 2000; Kippenberg/Stuckrad 2003.
} 
der Religionsgeschichte jedoch überhaupt nicht vor. Drittens wird gegenüber der Verwendung eines einheitlichen Religionsbegriffs eingewandt, dass es sich bei ihm um ein relativ spätes Produkt der europäischen Religionsgeschichte mit einem starken christentumsspezifischen und westlich-kulturellem Bias handelt ${ }^{4}$. In ihn seien Ablagerungen des abendländischen und christlich geprägten Denkens eingegangen, die seine universale Anwendbarkeit in hohem Maße einschränkten. Da der Religionsbegriff eine Erfindung der westlichen aufklärerisch geprägten Moderne sei, könne die Kategorie nicht mehr inhaltlich ernstgenommen werden, sondern nur noch dekonstruiert und auf ihre Funktion im populären Diskurs untersucht werden (Arnal 2000: 23, 30).

Die aufgelisteten Probleme bei der Erstellung einer universell verwendbaren Religionsdefinition halten die Religionshistoriker und Religionssoziologen in der Regel nicht davon ab, den Religionsbegriff dennoch zu verwenden (so auch Childester 1996: 259; Bergunder 2011: 5 und andere). Er ist ein Grundbegriff der Religionswissenschaft und der Religionssoziologie und zur Absteckung des Gegenstandsbereiches, mit dem diese Disziplinen befasst sind, unaufgebbar (Rüpke 2007: 31-32.). Ein Verzicht auf die Klärung dieses Grundbegriffs würde impliziten Vorannahmen und Werturteilen Tür und Tor öffnen und die empirische Analyse entgrenzen sowie willkürlichen Entscheidungen aussetzen. Natürlich sollte man die Bedeutung begrifflicher Festlegungen nicht überschätzen. Definitionen sichern nicht die Gegenstandsangemessenheit der Analyse, sondern stellen Konventionen dar, auf die man sich unter Forschern einigt und die stets auch anders ausfallen können. Als solche Übereinkünfte bilden sie allerdings die Grundlage der Arbeit, die die Verständigung über den Gegenstand und seine Beobachtung überhaupt erst ermöglicht. Die wissenschaftliche Analyse bedarf eindeutig definierter Kategorien, ohne deren Anwendung sie blind bleiben würde. ${ }^{5}$ Sie muss auf Defini-

\footnotetext{
${ }^{4}$ Vgl. Matthes 2005; Asad: 1993: 27-53; McCutcheon 1997; Fitzgerald 2000; Dubuisson 2003.

${ }^{5}$ So auch Graf (2004: 237): ,Wir brauchen allgemeine Begriffe, um das religiöse Feld strukturieren und von anderen Feldern abgrenzen zu können.
} 
tionen zurückgreifen, nicht nur um den zu untersuchenden Gegenstand inhaltlich zu bestimmen und von anderen Gegenständen unterscheiden zu können, sondern auch um Veränderungen im Gegenstandsfeld erfassen zu können. Die religionssoziologisch so relevante Frage nach dem gesellschaftlichen Bedeutungswandel von Religion und nach dem Wandel ihrer sozialen Funktionen lässt sich nur dann angemessen bearbeiten, wenn klar ist, was unter Religion zu verstehen ist; sonst besteht die Gefahr, dass der zeitliche Vergleich sich auf inhaltlich ungleiche Größen bezieht und Unvergleichliches miteinander verglichen wird. Die Erkenntnis von Varianz setzt einen einheitlichen Maßstab voraus.

Berechtigt ist das Argument, dass es unmöglich sein dürfte, mit der Angabe einer Religionsdefinition die Fülle religiöser Phänomene einzufangen. Der Begriff bleibt zwangsläufig hinter der Mannigfaltigkeit der Wirklichkeit zurück. Ob ein Begriff brauchbar ist oder nicht, kann nicht auf der theoretischen Ebene, sondern nur empirisch entschieden werden. Die Bewährung der Begriffsarbeit am empirischen Gegenstand bleibt insofern in der Tat eine prinzipiell unbeendbare Aufgabe.

Das schließt es allerdings nicht aus, Generalisierungen vorzunehmen, denn natürlich lassen sich unterschiedliche Phänomene einer abstrakten Kategorie zuordnen und unter sie subsumieren, ohne dass dadurch deren Besonderheiten nivelliert würden. Wenn der Buddhismus, das Christentum, der Islam, Scientology, Schamanismus und Bahai als konkrete Religionen behandelt werden, dann schließt das keineswegs aus, diese konkreten Religionen zu einem Begriff zusammenzufassen. Die Bedingung dafür besteht lediglich darin, dass es zwischen den Religionen etwas Gemeinsames gibt, das sie mit anderem verbindet, (genus proximum) und dass sie sich von anderen Phänomenen, etwa von Kunst, Philosophie oder Literatur, abgrenzen lassen (differentia specifica). Wenn

[...] Es geht $[\ldots]$ nicht um einen funktionalistischen, ubiquitär verwendbaren Begriff der Religion. Doch ist das kulturell Besondere oder Individuelle nur dann zu beschreiben, wenn uns allgemeinere Begriffe zur Verfügung stehen, mit denen sich die spezifische Differenz dieses Besonderen erfassen lässt.“ 
sich die Eigenart von Religion weder durch Angabe eines genus proximum noch durch Benennung von differentia specifica ausweisen ließe, dann müsste man den Begriff der Religion fallenlassen und stattdessen von etwas anderem reden, zum Beispiel von Kultur, Ideologie, Selbsttranszendierung, Gemeinschaft oder Kult. $\mathrm{Zu}$ einer solchen Auflösung des Religionsbegriffs etwa im Kulturbegriff tendieren auch tatsächlich einige Ansätze der neueren Religionswissenschaft, die in kritischer Reaktion auf die theologisch und phänomenologisch behauptete Unableitbarkeit von Religion (vgl. etwa Nathan Söderblom, Rudolf Otto, Friedrich Heiler) deren kulturelle Einbindung so stark betonen, dass sie ihren unterscheidbaren Status innerhalb der Kultur verliert (Sabbatucci 1988; Fitzgerald 2000; Debuisson 2003). Soll eine derartige Auflösung des Gegenstands der Religionswissenschaft und Religionssoziologie vermieden werden, muss mithin danach gefragt werden, ob sich Merkmale finden lassen, die allen Religionen gemeinsam sind und durch die sie sich zugleich von anderen Phänomenen unterscheiden lassen.

Was schließlich die Behauptung der Abhängigkeit unserer Erkenntnis von kultur- und gesellschaftsspezifischen Voraussetzungen angeht, so ist zunächst einzuräumen, dass es sich bei unseren wissenschaftlichen Analysen natürlich stets um Konstruktionen handelt, die von subjektiven Interessen und Vorurteilen, kulturellen Kontexten und sozialen Rahmenbedingungen beeinflusst sind und als solche dekonstruiert werden können. Daraus folgt allerdings nicht, dass unsere Begriffe und Kategorien ausschließlich von solchen im Rücken unseres Erkennens liegenden Voraussetzungen abhängig sind. Vielmehr müssen wir damit rechnen, dass wir unser Bild von der Wirklichkeit nicht nur eigenmächtig konstruieren, sondern dass in diesen Konstruktionsprozess der zu erkennende Gegenstand auch selber eingreift. Der radikale Konstruktivismus argumentiert nicht radikal genug. Sein Zweifel richtet sich nur auf die Erkennbarkeit der Wirklichkeit. Aber er müsste auch die Behauptung ihrer Unerkennbarkeit einbeziehen. Natürlich sind wir als Forschende an der Analyse unseres Gegenstandes immer auch produktiv beteiligt. Aber deshalb muss es sich bei unseren Erkenntnissen nicht um außerempirische Fiktionen handeln. 
Der Erkenntnisprozess ist eben ein zweiseitiger Prozess, in dem das Subjekt nicht willkürlich fingieren kann, was das Objekt ausmachen soll, sondern in dem das Objekt auch selbst zum Subjekt werden kann und sich gegenüber den Übermächtigungsversuchen der anderen Seite als widerständig zu erweisen vermag. Vielleicht täuschen wir uns in vielen unserer Wirklichkeitsannahmen gar nicht. Wir wissen zwar nicht, ob unsere Erkenntnis wahr ist. Aber wir wissen auch nicht, dass sie unwahr ist. Selbst wenn unsere Kategorien von Vorannahmen geprägt sind, müssen sie nicht grundsätzlich falsch sein; schließlich stammen unsere Vorannahmen ja wesentlich selber aus jener Wirklichkeit, um deren Erkenntnis es uns geht.

\section{Unterschiedliche Religionsbegriffe}

Um die Vor- und Nachteile unterschiedlicher Religionsdefinitionen kennenzulernen, seien im Folgenden einige gebräuchliche Definitionsmethoden vorgestellt.

\subsection{Substanzielle Definitionen}

Eine gebräuchliche Herangehensweise an das Problem der Religionsdefinition besteht in dem Entwurf von inhaltlichen oder substanziellen Religionsdefinitionen. In diesen Fällen erfolgt die Definition der Religion durch Angabe ihrer wichtigsten Merkmale, etwa durch den Versuch, die Vielfalt religiöser Erscheinungsformen durch Angabe des Bezugsgegenstandes, auf den sich Religionen richten, zu bändigen und Religionen durch den Bezug auf Gott oder Götter, durch die Verehrung von höheren geistigen Wesen oder den Glauben an einen oder mehrere Götter zu charakterisieren $^{6}$. Der Vorzug einer solchen auf den Gottesbezug abhebenden Religionsdefinition besteht in ihrer Nähe zum religionshistorischen Material sowie in ihrem engen Bezug auf das Selbstverständnis der Religionsangehörigen. Ihr Problem liegt darin, dass es einige Religionsformen gibt, etwa den frühen Buddhismus, die Rituale des jungpaläolithischen höheren Jägertums sowie neuere

\footnotetext{
${ }^{6}$ Vgl, Tylor 1871: 383-384; Widengren 1969: 4, 46-47; Pettazoni 1960; Lanczkowski 1980: 23-24.
} 
Religionsvorstellungen, in denen der Gottesbezug keine oder nur eine untergeordnete Rolle spielt.

Anstelle des Gottesbegriffs verwenden einige Religionswissenschaftler daher die Kategorie des „Heiligen“ (zentral noch immer: Otto 1917) und erklären, dass in allen Religionen die Verehrung eines wie auch immer gearteten „Heiligen“ im Zentrum stehe. Mit einer solchen Begrifflichkeit macht sich der Religionsforscher jedoch abhängig vom Selbstverständnis der Religionsangehörigen, denn was jeweils als ,heilig“ gilt, kann nur von diesem her bestimmt werden und vermag daher ganz unterschiedlich auszufallen. Auch die Verehrung von Nation, Familie, Freiheit oder Geld kann dann als Religion erscheinen, wenn diese Werte und Institutionen als heilig verehrt werden. Damit verliert der Begriff allerdings seine Trennschärfe. ${ }^{7}$

Häufig wird deshalb weder der Begriff Gottes noch der des Heiligen, sondern der des Übernatürlichen in den Mittelpunkt der Religionsdefinition gerückt (Heiler 1962: 13). Aber auch hier sind wir wieder mit dem Problem konfrontiert, dass der angegebene Bezugsgegenstand nicht eindeutig nur auf Religion und Religiöses applizierbar ist. Ein Bezug zum Übernatürlichen und Außergewöhnlichen kann auch für die Kunst, die Literatur, den Tanz, die Ekstase oder das Spiel behauptet werden. In all diesen Formen hat es der Mensch mit einer Ausweitung seiner alltäglich gegebenen Erfahrungsmöglichkeiten und einer Überschreitung eingewöhnter

\footnotetext{
${ }^{7}$ Das religiöse Selbstverständnis zum Maßstab dessen zu machen, was analytisch unter Religion zu verstehen ist, wirft ein doppeltes Problem auf, denn dann müssen nicht nur Phänomene als religiös behandelt werden, die von Religion deutlich unterschieden sind, etwa die Begeisterung für Fußball, Erfolg oder Geld, sondern dann muss auch religiösen Phänomenen wie etwa dem Freidenkertum oder der Naturmystik, die von den Akteuren vielfach nicht religiös gedeutet werden, der Religionsstatus abgesprochen werden. Die Begeisterung für Fußball zum Beispiel lässt sich aber von religiösen Vorstellungen und Praktiken durchaus unterscheiden, da es sich beim Gegenstand der Begeisterung nicht um etwas Transzendentes handelt, während es umgekehrt durchaus sinnvoll sein kann, die Vorstellungen, Weltdeutungen und Praktiken von Freidenkern zum Beispiel als religiöse zu interpretieren, obschon sie diese als wissenschaftliche verstanden wissen wollen.
} 
Grenzen zu tun. Lässt sich schon dann von Religion sprechen, wenn der Mensch seine Alltagswelt transzendiert?

Im phänomenologischen Ansatz von Alfred Schütz (1971), der von Peter L. Berger und Thomas Luckmann (1966) aufgenommen wurde, wird zwischen verschiedenen Sinnprovinzen wie etwa der Erlebniswelt des Theaters, der Welt der theoretischen Spekulation, der Traumwelt oder auch der Erfahrungswelt der Religion und der Alltagswelt als der dichtesten und obersten Realitätszone unterschieden. In die Alltagswelt kehre der Mensch nach seinen Ausflügen in die verschiedenen Sinnprovinzen immer wieder zurück. Wenn der Mensch die alltägliche Welt seiner Verrichtungen überschreitet, begibt er sich dem phänomenologischen Ansatz zufolge also nicht notwendigerweise in den Erfahrungsbereich der Religion. Das Verlassen der Welt des Alltäglichen und des unmittelbar Zugänglichen vollzieht sich nach diesem Ansatz vielmehr auch im Besuch eines Theaters, im Traum, ja selbst in der soziologischen Theoriearbeit, ohne dass all diese Grenzüberschreitungen bereits als religiös klassifiziert werden können.

Im Lichte der Argumentation des phänomenologischen Ansatzes erweist sich auch der Begriff der Selbsttranszendenz ${ }^{8}$ zur Kennzeichnung religiöser Erfahrungen als nicht spezifisch genug. Selbsttranszendenz ist letztlich ein ordinäres Merkmal jeder menschlichen Erfahrung, sofern es sich dabei um eine Erfahrung von etwas anderem handelt, als es das Individuum selbst ist. Erfahrungen dieses Typs als religiös zu qualifizieren hieße, jeder gegenständlichen Erfahrung einen religiösen Charakter zuzusprechen. Mit der Festlegung der Religion auf ihre Erfahrungsdimension würde darüber hinaus von weiteren Merkmalen, die für Religionen typisch sind, abgesehen, etwa von ihrem Traditionsbezug

\footnotetext{
${ }^{8}$ Das Konzept der Selbsttranszendenz wird von Hans Joas (2004: 43) vertreten. Allerdings weist JOAS darauf hin, dass die Erfahrung des Ergriffenwerdens und des Sich-Hingebens, wie sie sich im Falle der Selbsttranszendenz einstellt, der Auslegung bedarf, um Eindeutigkeit zu gewinnen. In dieser greift der Mensch auf kulturelle Deutungsvorräte und Traditionen zurück, und es mag sein, dass dadurch Erlebnisse der Selbsttranszendenz als religiöse erkennbar werden. Zum Verhältnis von religiösem Erlebnis und religiöser Erfahrung vgl. Krech (1998: 478).
} 
oder ihrer rituellen Verankerung. Substanzielle Religionsdefinitionen werfen offenbar also das Problem auf, dass sie auf der einen Seite Merkmale (zum Beispiel den Gottesbegriff) als typisch religiös identifizieren, die nicht bei allen religiösen Phänomenen anzutreffen sind (zum Beispiel nicht bei neueren Formen der Spiritualitäts- und New-Age-Religiosität) und auf der anderen Seite Charakteristika für Religion ausmachen (das Übernatürliche, Außergewöhnliche), die sich auch in nicht-religiösen Phänomenen finden lassen (zum Beispiel in der Kunst oder im Spiel). ${ }^{9}$ Darüber hinaus sind sie aber auch nicht davor gefeit, Kennzeichen zu vernachlässigen, die für Religion typisch sind (das Ritual, die Gemeinschaft, die Tradition). ${ }^{10}$ Die Aufgabe substanzieller Religi-

\footnotetext{
${ }^{9}$ Da der Bezugsgegenstand der Religion schwer greifbar ist und seine Bestimmung entweder zu weit oder zu eng ausfällt, neigen manche Religionswissenschaftler dazu, die Erstellung einer Religionsdefinition nicht auf dem Wege der Bestimmung des religiösen Bezugsgegenstandes, sondern über die Art und Weise des religiösen Bezugs auf einen Gegenstand vorzunehmen (Waardenburg 1984: 238-255). Um Religion handelt es sich demzufolge dann, wenn etwas für den Beteiligten absolute Gültigkeit besitzt. Mit dem Anspruch auf absolute Gültigkeit kann das menschliche Tun und Trachten aber auch ausgestattet sein, wenn es sich auf sportliche Dinge, auf Sexualität, Politik oder Wissenschaft richtet. Sakralisierung ist in allen Bereichen des menschlichen Handelns möglich und insofern kein genuines Merkmal von Religion.

${ }^{10}$ Eine Argumentation wie die hier vorgetragene ist in mancherlei Hinsicht nicht unproblematisch. Sie geht davon aus, dass sich Religion identifizieren und etwa von Kunst, Wissenschaft oder Liebe unterscheiden lässt. Schon dies könnte bezweifelt werden, da diese Wirklichkeitsbereiche unter bestimmten Gesichtspunkten, etwa dem der Selbsttranszendenz, auch als gleichartig behandelt werden könnten. Dann wäre freilich auch wissenschaftliches Nachdenken und das Erlebnis einer Theateraufführung als religiös einzuordnen. Außerdem könnte eingewandt werden, dass die Kritik an den unterschiedlichen vorgestellten Religionsbegriffen immer schon eine Kenntnis von dem voraussetzt, was Religion ist. Dieser Einwand ist berechtigt. Ein voraussetzungsloses Definieren kann es nicht geben. Beim Definieren muss ein Vorwissen in Anspruch genommen werden, das natürlich fragmentiert und einseitig ist, von dem her aber ein gehaltvoller Begriff des in Frage stehenden Gegenstandes einzig entworfen werden kann. Auch wenn Begriffsbestimmungen so stets arbiträr bleiben, sind sie jedoch nicht sinnlos. Mit ihnen können Phänomene der Wirklichkeit identifiziert und von anderen abgehoben, also beobachtbar gemacht werden. Deren empirische Analyse kann dazu dienen herauszufinden, inwieweit die Begriffsbestimmung mit dem Phänomen übereinstimmt und an welchen Stellen die
} 
onsdefinitionen besteht folglich darin - hier nehmen wir oben bereits angestellte Überlegungen noch einmal auf -, diejenigen Merkmale zu identifizieren, die Religionen mit anderen Phänomenen gemeinsam haben (genus proximum) und die zugleich nur ihnen eigen sind, sie also von anderen Phänomenen unterscheiden (differentia specifica).

Eine neuere Religionsdefinition, die man als substanziell kennzeichnen kann und die Stärken und Schwächen mit anderen substanziellen Ansätzen teilt, stammt von Martin Riesebrodt. In Abgrenzung von funktionalistischen Definitionen, die den vermeintlichen Beitrag von Religion für die Konstitution und Reproduktion der Gesellschaft erklären, aber keinen Beitrag zum Verständnis von Religion leisten, wählt Riesebrodt (2007: 110) einen handlungstheoretischen Ansatz, der am Sinn religiösen Handelns orientiert ist. Die spezifische Sinngebung des religiösen Handelns liegt, so Riesebrodt, , in ihrem Bezug auf persönliche oder unpersönliche übermenschliche Mächte, d.h. Mächte, die kontrollieren oder beeinflussen, was sich menschlicher Kontrolle entzieht" (ebd.: 108). Durch religiöse Praktiken nimmt der Mensch Kontakt zu diesen Mächten auf oder gewinnt Zugang zu ihnen. Interventionistische Praktiken wie etwa Gebete, magische Formeln, Opfer, Orakel oder Gesang, durch die die Menschen die höheren Mächte zu manipulieren, zu beeinflussen oder gar mit ihnen zu kommunizieren versuchen, stellen den Kern jeglicher Religion dar (ebd.: 2000: 41). Religion wird von Riesebrodt (2007: 120, 127, 117) primär nicht als Weltbild oder Symbol- und Glaubenssystem, als Moral oder Ethik und auch nicht als Theologie oder Philoso-

entworfenen Klassifikationen zusammenbrechen (Berger 2014: 25). Die Entwicklung kategorialer Bestimmungen ist ein Hin- und Hergehen zwischen begrifflicher Abstraktion und empirischer Überprüfung, empirischer Anregung und theoretischer Systematisierung. Einen Fixpunkt kann es nicht geben, weder in der Theorie noch in der Empirie. Vielmehr handelt es sich hier um einen unabschließbaren Prozess. Wenn dieser Prozess um der Pragmatik der wissenschaftlichen Arbeit willen an einem bestimmten Punkt gestoppt wird, so kann es sich bei der erzielten Übereinkunft lediglich um ein vorläufiges Ergebnis handeln, das angesichts neuer Einsichten jederzeit wieder aufgekündigt zu werden vermag. 
phie gefasst, sondern als eine sozial konstituierte interventionistische Praxis, die darauf abzielt, durch den Kontakt mit übermenschlichen Mächten, Einfluss auf dasjenige zu nehmen, das sich menschlicher Kontrolle entzieht und auf diese Weise „Krisen (Risiken, Gefahren) vorzubeugen oder diese zu bewältigen, wenn sie eingetreten sind" (2000: 42). Das Heilsversprechen der Religion, ihre Kapazität zur Abwehr von Unheil und zur Krisenbewältigung stellt dabei keine latente Funktion religiöser Praktiken dar, sondern den in sie eingeschriebenen Sinn (2007: 132).

Mit einem solchen Religionsbegriff ist als das Zentrum der Religion der kultische Vollzug und das rituelle Handeln bestimmt, während religiösen Diskursen, religiösen Deutungen und Theologien sowie verhaltensregulierenden Vorschriften allenfalls ein sekundärer Status zukommt und subjektive religiöse Erfahrungen für die Definition von Religion überhaupt keine Rolle spielen. Abgesehen ist damit von Formen einer passiven, mystischen Religiosität, die das Ruhen in Gott anstrebt, sowie von Formen einer deistischen Religiosität, die Gott als Grund der Welt voraussetzt, ohne auf ihn interventionistisch Einfluss nehmen zu wollen oder diesen Einfluss auch nur für möglich zu halten. Zugleich weist der Religionsbegriff Riesebrodts eine auffällige Nähe zu einem modernen Technikverständnis auf, das davon ausgeht, durch Steigerung technischer Kontroll- und Steuerungsmöglichkeiten die Beherrschung von Natur, Mensch und Gesellschaft erhöhen und Krisen vorbeugen zu können. Gott wird von Riesebrodt als ein ethisches, interventionistisches höheres Wesen behandelt, obschon er zuweilen auch als übermächtiges Wesen im Hintergrund, als Deus otiosus oder sogar als Deus absconditus, der sich dem menschlichen Zugriff entzieht, aufgefasst werden kann (vgl. Eliade 1954: 71ff.), und Religion wird von ihm als eine Art technisch-magische Praxis definiert, womit nicht nur ihr Erfahrungscharakter hintangestellt wird, sondern auch eine starke Überschneidung zu säkularen technischen Praktiken entsteht. Wieder erweist sich die entworfene Religionsdefinition auf der einen Seite als nicht umfassend genug und auf der anderen als nicht spezifisch genug. $\mathrm{Zu}-$ gleich sind mit ihr aber doch auch zentrale Merkmale von Religion erfasst. 


\subsection{Funktionalistische Religionsdefinitionen}

Um den Problemen zu entgehen, die aus einer Definitionsmethode entstehen, die bei den inhaltlichen Annahmen von Religionen, zum Beispiel beim Bezugsgegenstand der Religion ansetzen, bestimmt die funktionale Herangehensweise Religion nicht aus sich selbst heraus, sondern von dem her, was sie nicht ist, und geht von den gesellschaftlichen oder individuellen Zusammenhängen aus, in denen sie steht. Die funktionale Methode bezieht Religion auf ein Problem, das mit dieser gelöst ist (Luhmann 1977: 9f.), zum Beispiel auf das Problem des gesellschaftlichen Zusammenhalts, und bestimmt die Leistung, die die Religion zur Lösung dieses Problems erbringt. Während es in substanziellen Definitionen darum geht festzustellen, was Religion ist, kommt es bei funktionalen Definitionen darauf an, zu bestimmen, was Religion leistet und bewirkt (Dobbelaere/Lauwers 1974: 536). Unter funktionalen Gesichtspunkten könnte man dann zum Beispiel sagen, Religion zeichne sich dadurch aus, dass sie die Integration einer Gruppe, einer Gemeinschaft oder einer Gesellschaft gewährleiste.

Eine charakteristische funktionalistische Religionsdefinition findet sich bei Pippa Norris und Ronald Inglehart. ${ }^{11}$ Für Norris und Inglehart (2004) wird die Bedeutung, die Religion in einer

\footnotetext{
${ }^{11}$ Die oft als funktionalistisch verstandene und von ihm auch als funktionalistisch angebotene Religionsdefinition von Thomas Luckmann ist im strengen Sinne keine funktionalistische - so schon Marquard (1985: 42) sowie Spaemann (1985: 48). Wenn Religion nach Luckmann (1985: 27; 1991: 108) dasjenige ist, was den Menschen „zum Menschen werden lässt“, dann handelt es sich bei ihr um einen unausweichlichen Bestandteil der conditio humana. Welches Bezugsproblem mit ihr gelöst sein soll, bleibt unklar. Allenfalls ließe sich sagen, Religion komme nach Luckmann eine Sozialisierungsfunktion zu. Besteht das Problem, das durch Religion behandelt wird, dann darin, dass der Mensch eigentlich kein soziales Wesen ist und durch Religion dazu erst gemacht wird? In diese Richtung weist die schwer auflösbare Formulierung Luckmanns, die Funktion der Religion bestehe in der „Vergesellschaftung des Umgangs mit Transzendenz“ (1985: 26). Gibt es einen nicht vergesellschafteten Umgang mit Transzendenz? Dies stände im Widerspruch zu der von Luckmann (1991: 108) gleichfalls vertretenen Annahme, dass „Menschlichkeit“ dem Menschen ,durch die jeweiligen gesellschaftlichen Formen der Religion vorgegeben" sei.
} 
Gesellschaft besitzt, vor allem durch das Gefühl der existenziellen Sicherheit und der Verletzbarkeit durch physische, gesellschaftliche und personale Risiken bestimmt. In Gesellschaften, die stärker existenziellen Risiken ausgesetzt sind, ist der Bedarf an Religion größer als in Gesellschaften, in denen ein höherer Grad an existenzieller Sicherheit existiert. Existenzielle Sicherheit meint zum einen Freiheit von Naturkatastrophen wie Flut, Erdbeben, Dürre und Tornados, zum anderen Freiheit von sozial produzierten Risiken und Gefahren wie Krieg, Menschenrechtsverletzungen, Armut und sozialer Ungleichheit. In dem Maße, wie Gesellschaften den Frieden sichern, sich Zugang zu ausreichenden Nahrungsmitteln verschaffen, ihr Gesundheitssystem verbessern, Einkommenszuwächse garantieren und soziale Ungleichheiten abbauen, steige das Niveau empfundener existenzieller Sicherheit, während der Bedarf für religiöse Werte, Glaubenssysteme und Praktiken abnehme. Ausschlaggebend für die Erfassung von Religion ist mithin die Analyse ihres Umgangs mit dem Problem der existenziellen Unsicherheit. Dies ist das Bezugsproblem der Religion.

Gegenüber diesem Ansatz ist einzuwenden, dass Religion es nicht nur mit Erfahrungen existenzieller Unsicherheiten zu tun hat, sondern auch mit der Steigerung individueller Erlebnismöglichkeiten, mit Entgrenzungserfahrungen und Erlebnissen der Verschmelzung mit dem Anderen, mit der Legitimation politischer Ordnung oder der Motivation ordnungszerstörender und terroristischer Akte. Außerdem kann auf Erfahrungen existenzieller Unsicherheiten nicht nur durch die Gewährung religiös begründeter Sicherheiten, sondern auch durch den Aufbau wohlfahrtsstaatlicher Einrichtungen, die Konstitution familiärer Solidaritätsnetzwerke, durch Wohlstandsanhebung, Entwicklung von Versicherungssystemen oder Verbesserung der medizinischen Betreuung reagiert werden. Schließlich könnte eine Reaktion auf dieses Problem auch ausfallen und die Frage nach dem angemessenen Umgang mit existenziellen Unsicherheiten unbeantwortet bleiben. Das Angebot von religiösen Deutungssystemen und Praktiken ist nur eine Möglichkeit des Umgangs mit dem Unsicherheitsproblem unter vielen. Ein funktionaler Bedarf für Religion bringt nicht zwangsläufig auch die ihm entsprechenden Religionsformen her- 
vor. Sofern funktionale Ansätze so wie der von Norris und Inglehart von dem Bedarf für Religion umstandslos auf ihre gesellschaftliche Bedeutung schließen und das Problem funktionaler Äquivalente nicht eigens thematisieren, neigen sie dazu, ein verkürztes Bild von den Bedingungsfaktoren der gesellschaftlichen Relevanz von Religion zu zeichnen.

Gegenüber dem Gebrauch der funktionalen Definitionsmethode sind also vor allem zwei Einwände zu machen. Der eine Einwand bezieht sich darauf, dass die funktionale Methode ihr Objektfeld zu weit fasst und auch Phänomene in ihren Erfassungsbereich mit aufnimmt, die selbst bei einem weiten Religionsverständnis nicht als Religion zu begreifen sind. ${ }^{12}$ Auf Erfahrungen existenzieller Unsicherheiten kann auch durch den Aufbau wohlfahrtsstaatlicher Einrichtungen, die Konstitution familiärer Solidaritätsnetzwerke, durch Wohlstandsanhebung oder die Entwicklung von Versicherungssystemen reagiert werden. Die Bearbeitung von Gefühlen existenzieller Unsicherheit stellt kein Spezifikum religiöser Deutungssysteme und Praktiken dar. Wenn der Bezug auf das Unsicherheitsproblem zum Bestimmungsmerkmal von Religion gemacht wird, dann können auch nichtreligiöse Institutionen und Inhalte religiöse Funktionen wahrnehmen und als Religion firmieren, wodurch die Definition ihre Trennschärfe verliert.

Der andere Einwand besteht darin, dass das ausgemachte Bezugsproblem, etwa das Unsicherheitsproblem, vielleicht nicht präzise gefasst ist und viele religiösen Inhalte und Formen gar nicht zu umgreifen vermag. Hat es Religion nicht auch mit der Steigerung individueller Erlebnismöglichkeiten, mit Entgrenzungserfahrungen und Erlebnissen der Verschmelzung mit dem Anderen, mit der Legitimation politischer Ordnung oder der Motivation ordnungszerstörender und terroristischer Akte zu tun und nicht nur mit Erfahrungen existenzieller Unsicherheit? Ist mit der Zuwei-

\footnotetext{
${ }^{12}$ Das Problem der Allgemeinheit und Abgrenzbarkeit der funktionalen Religionsdefinition ist breit diskutiert. Vgl. Goody 1961; Spiro 1966: bes. 95-96.; Matthes 1967: bes. 19-20; Berger 1973; Berger 1974; Dobbelaere/Lauwers 1974; Luhmann 1977; Seiwert 1981; Wuthnow 1988; Kaufmann 1989: 15ff.; Lambert 1991; Tyrell 1996: 440ff.; Figl 2003: 67-69; Stausberg 2009; Hock 2011: 16-17.
} 
sung der Funktion der Bewältigung existenzieller Unsicherheit das Spezifikum religiöser Praktiken, Überzeugungen und Identitäten getroffen oder nicht möglicherweise verfehlt?

Bourdieu schlägt als Ausweg aus den Inkonsistenzen und Inkommensurabilitäten bei der Bestimmung eines allgemeingültigen Religionsbegriffes vor, von den Kämpfen auszugehen, die die religiösen Akteure zur Bestimmung des religiösen Feldes selbst austragen (Bourdieu 2009: 243). ${ }^{13}$ Was Religion sei, lasse sich nicht durch eine „vorgängige Definition“ positivistisch festlegen, denn das religiöse Feld sei der Austragungsort des Kampfes um die Definition und damit um die Abgrenzung von Kompetenzen (ebd.: 244). In Konkurrenz zu diesem hermeneutischen Prinzip steht allerdings der Vorschlag Bourdieus, sich nicht auf die Definition der religiösen Akteure und Experten zu verlassen, sondern Grenzverschiebungen $\mathrm{zu}$ beobachten, die darauf beruhen, dass $\mathrm{zu}$ den Seelsorgern und Geistlichen ganz neue Experten hinzutreten, die sich mit etwas befassen, das - so offenbar die Annahme Bourdieus - in den Zuständigkeitsbereich „der Geistlichen alten Schlags“ fällt: die „Behandlung des Seelenheils“ (ebd.: 246). Diese Aufgabe übernähmen nunmehr Psychoanalytiker, Soziologen, Sozialarbeiter und andere säkulare Experten, wodurch diese in den Rang religiöser Experten einrückten. Dass Bourdieu Psychoanalytiker, Soziologen und Sozialarbeiter als neue religiöse Experten behandelt, ist allerdings der Tatsache geschuldet, dass seine Argumentation dann doch wieder von einer vorgängigen Definition dessen, womit es Religion zu tun hat, abhängig ist: von der Bestimmung des religiösen Bezugsproblems als Umgang mit dem Seelenheil. Diese vorgängige Religionsdefinition ist insofern problematisch, als nicht angegeben wird und nicht angegeben werden kann, warum es in der Religion gerade um das Seelenheil - und nicht etwa

\footnotetext{
${ }^{13}$ Der Versuch, das Problem der Religionsdefinition durch Rückgang auf das Selbstverständnis der Gläubigen zu lösen, ist nicht ungebräuchlich. Religion ist dann alles, was die Religionsangehörigen darunter verstehen. Vgl. etwa Hölscher (2005: 15), der als religiös ,all diejenigen historischen Erscheinungen“ behandelt, „die in den Quellen so bezeichnet worden sind“ (Hölscher 2005: 14). Bei einer solchen Orientierung am Selbstverständnis kommt es allerdings leicht zu einer „übergroßen Fülle an Kategorien bzw. Typen“ (Schäfer 2009: 556).
} 
die Legitimation politischer Ordnung, die Fundierung letztgewisser Wahrheit, die Interpretation der Welt im Ganzen oder die Heilung des Körpers - gehen soll. Und sie ist insofern problematisch, als es für den Umgang mit Seelenproblemen natürlich auch andere Sinnformen als die religiösen gibt, etwa Psychotherapie, Philosophie, Kunst, Literatur oder Körpertechnik - Sinnformen, die damit ja noch nicht in den Erfassungsbereich des Religionsbegriffes fallen. Es handelt sich bei dem Vorschlag Bourdieus mithin um eine funktionale Religionsbestimmung, die noch nicht einmal ein Bewusstsein darüber entwickelt, dass sie eine vorgängig vorgenommene funktionale Religionsdefinition darstellt. Und wie alle funktionalen Religionsdefinitionen vermag auch sie nicht anzugeben, warum gerade der ausgemachte Problemgesichtspunkt das religiöse Bezugsproblem sein soll und warum es nicht auch andere als religiöse Lösungen auf dieses Bezugsproblem geben kann.

Die inneren Widersprüche im Bourdieuschen Ansatz und in den funktionalen Religionsdefinitionen insgesamt laufen auf zwei Probleme $\mathrm{zu}$, denen sich jeder Versuch einer allgemeingültigen Religionsdefinition stellen muss: auf die Frage, wie sich wissenschaftliche Außenperspektive und religionsinterne Binnenperspektive zueinander verhalten, und auf die Frage, in welchem Verhältnis funktionale Gesichtspunkte und inhaltliche Bestimmungsgründe stehen. Auf beide Probleme wollen wir in unserem eigenen Definitionsvorschlag, der unten unter 3. entfaltet werden soll, durch eine Kombination funktionalistischer und substanzieller Argumente sowie durch eine Kombination von Außen- und Innenperspektive reagieren.

Die bourdieusche Argumentation weist allerdings noch ein weiteres Problem auf, das der Bearbeitung bedarf: die Bestimmung des Verhältnisses des wissenschaftlichen Religionsbegriffs $\mathrm{zu}$ einem unerklärten, gleichwohl aber implizit vorausgesetzten alltagsweltlichen Religionsverständnis, das in den wissenschaftlichen Definitionsversuchen anscheinend immer mitläuft. Wenn Bourdieu Psychoanalytiker, Soziologen und Sozialarbeiter, die sich mit dem Seelenheil beschäftigen, als religiöse Experten anspricht, dann hat er offenbar eine bestimmte Vorstellung von Religion im Kopf, die er seinen soziologischen Überlegungen $\mathrm{zu}$ 
Grunde legt. Auch die hier angestellten Überlegungen haben sich immer wieder auf ein nicht weiter expliziertes Religionsverständnis bezogen und daran die ins Spiel gebrachten Begriffsvorschläge gemessen. So war etwa die Rede davon, dass die vorgeschlagenen Religionsdefinitionen Merkmale als religiös behandeln, die nicht bei allen religiösen Phänomenen anzutreffen sind bzw. sich auch bei nicht-religiösen Phänomenen finden lassen, oder dass sie Merkmale in ihren Gegenstandsbereich aufnehmen, die selbst bei einem weiten Religionsverständnis nicht als religiös anzusehen seien. Macht, wer so argumentiert, die wissenschaftliche Analyse nicht vom außerwissenschaftlichen Diskurs über Religion, von einem vorausgesetzten, aber nicht explizierten Vorverständnis von Religion, also letztendlich vom Alltagsverständnis von Religion abhängig? Tatsächlich ist die wissenschaftliche Bestimmung des Religionsbegriffs unausweichlich an das Alltagsverständnis von Religion zurückgebunden. Mit dem aus dieser Einsicht resultierenden Versuch, den Religionsbegriff vom Alltagsdiskurs über Religion her zu bestimmen, wollen wir uns in unserer Auseinandersetzung mit vorliegenden Religionsbegriffen abschließend beschäftigen.

\subsection{Religion als Diskurs}

Das Alltagsverständnis von Religion zum Ausgangspunkt der Religionsbestimmung zu machen, wird seit einigen Jahren von mehr und mehr Religionswissenschaftlern und Religionssoziologen gefordert. Arthur Greil (2009: 148) etwa meint, Religion stelle weniger eine Entität als eine Diskurskategorie dar, deren Bedeutung in sozialen Interaktionen ausgehandelt werde. Auch Michael Bergunder (2011: 16) vertritt die Auffassung, dass die Religionswissenschaft nicht autonom über ihren Gegenstand zu verfügen vermag. Die klassischen Religionsdefinitionen seien heute diskreditiert; sie bezögen ihre Plausibilität einzig aus ihrem Bezug auf die Alltagsdiskurse über Religion. Daher mache ,faktisch“ das unerklärte, alltägliche Religionsverständnis ,,den Gegenstand der Religionswissenschaft aus" (Bergunder 2011: 17). Ebenso favorisieren auch Religionssoziologen wie Karl Gabriel (1993) oder Historiker wie Lucian Hölscher (2005: 11), der in seiner Geschichte des Pro- 
testantismus von „der religiösen Praxis und den religiösen Vorstellungen der Gläubigen“" ausgeht, einen diskurstheoretischen Religionsbegriff. Ein solches Religionsverständnis vertrat vor etlichen Jahrzehnten übrigens auch bereits Joachim Matthes (1992: 129), der den objektiven Gehalt einer soziologischen Bestimmung des Religionsbegriffes aufgrund des in ihm mitschwingenden westlich-christlichen Erbes bestritt und Religion als einen „diskursiven Tatbestand" verstand.

Wie lässt sich die Spezifik der diskurstheoretischen Erfassung des Gegenstandes der Religionsforschung bestimmen? Es handelt sich um eine Argumentation in drei analytischen Schritten, die hier anhand des Ansatzes von Michael Bergunder (2011) erläutert werden sollen. Der erste Schritt besteht in der Kritik an jeder Form einer gegenstandsbezogenen Religionsdefinition. Gegenständliche Religionsdefinitionen seien zum Scheitern verurteilt, da sich zwischen Wort, Begriff und Sache nicht klar unterscheiden lasse (Bergunder 2011: 26). Unseren Begriffen, so erklärt Bergunder unter Aufnahme der bekannten Argumentation von Jacques Derrida, liege nicht eine ,invariante Referenz, ein Eigentliches außerhalb des sprachlichen Zeichensystems, in dem sie ausgedrückt werden, zugrunde“ (ebd.: 29). Der Referenzcharakter von Begriffen wie etwa dem der Religion bleibe unklar. Deshalb müsse die Vorstellung eines ,transzendentalen Signifikats [...], das angeblich die Bedeutung des Signifikanten garantiert," aufgegeben werden (ebd.). Die Bedeutung sprachlicher Zeichen sei nicht in ihnen selbst gegeben, sondern ergebe sich lediglich aus ihrer Differenz zu anderen Zeichen. Ihre Bestimmtheit erhielten sie mithin nicht aus ihrer Referenz auf ein vorgestelltes Außen, sondern nur durch Bezug auf andere Zeichen, so dass sich das Spiel des Bezeichnens ins Unendliche ausdehne, aber niemals an einem Ding seinen gegenständlichen Abschluss finde.

Nachdem auf diese Weise allen begrifflichen Bestimmungen der empirische Gehalt abgesprochen wurde, geht es in einem zweiten Schritt um die analytische Aufhellung des ins Unendliche erweiterten Spiels des Bezeichnens. Es ist klar, dass die Technik des Aufhellens selbst Bestandteil des unendlichen diskursiven Spiels ist und die religionswissenschaftliche Beobachtung inso- 
fern selbst zur Religionsgeschichte gehört (Bergunder 2011: 47). Zwischen wissenschaftlicher Beschäftigung mit Religion und Alltagsdiskurs über Religion besteht kein Graben; vielmehr bilden wissenschaftliche Analyse von Religion, journalistische Berichterstattung über Religion, politische Rede zur Religion, Alltagsgespräche über Religion ein ins Unendliche ausgreifendes diskursives Netzwerk ohne außerdiskursiver Referenz (ebd.: 44-45.). Die Interaktivität dieses Diskurses kann nicht überschritten werden, auch nicht durch Einnahme einer wissenschaftlichen Beobachterperspektive. In dieser Beschränkung liegt aber zugleich auch ihr Vorzug, denn jede Bedeutungsfixierung, auch die wissenschaftliche, wird damit als eine sozial ausgehandelte Grenzziehung erkannt (ebd.: 34). Die Unterbrechung der unendlichen Interdependenzen durch solche Bedeutungsfixierungen stellen daher keine Rekonstruktion der Wirklichkeit, sondern einen neuschöpferischen Akt dar, der die zu beschreibende Wirklichkeit überhaupt erst hervorbringt und im Kampf um konkurrierende Deutungen erschafft (35-38). Die Erhellung des Diskurses ist folglich keine bloße Wiederholung des Diskurses, sondern setzt zwangsläufig eine Differenz.

Indem diese Differenz im Laufe der Geschichte verschleiert wird und dadurch der Eindruck kraftvoller Wiederholungen entsteht, bilden sich ,reifizierte, identifikatorische Allgemeinbegrif-

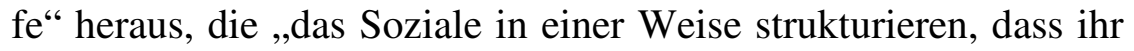
umkämpfter Charakter nicht mehr deutlich wird" (Bergunder 2011: 41). Im Anschluss an Laclau und Butler spricht Bergunder von Sedimentierungen (ebd.: 38, 41, 43). Diese in der Geschichte aufgehäuften Ablagerungen können nachgezeichnet und genealogisch erschlossen werden. Ziel dieser Genealogie ist es natürlich nicht, den Ursprung und die Einheit der Geschichte zu erfassen, wohl aber kann im Rückgang vom heutigen Alltagsverständnis von Religion „das gegenwärtige synchrone Netzwerk ,Religion““ auf frühere Religionsnetzwerke bezogen und der Alltagsdiskurs über Religion auf seine Kontinuität und Diskontinuität hin untersucht werden (ebd.: 44).

Was ist mit einer solchen an Foucault angelehnten genealogischen Diskursanalyse analytisch gewonnen? Man muss nüchtern 
konstatieren: nicht allzu viel. Der diskursanalytische Ansatz löst das erkenntnistheoretische Problem der Unüberschreitbarkeit sprachlicher Zeichensysteme und Bezeichnungsprozesse und damit der unüberwindbaren Differenz von Erkenntnis und Sache in keiner Weise. Natürlich bewegt sich die Diskursanalyse selbst im Medium des Diskurses. Wenn allerdings eine genealogische Erschließung des Religionsdiskurses vorgenommen werden soll, dann ist eben dieser Diskurs das transzendentale Signifikat, das Außen, das ebenso unerreichbar ist wie in begrifflichen Bestimmungen die gemeinte historisch-soziale Realität. Wer das transzendentale Signifikat aufgibt, hat damit die Spaltung von Erkenntnis und Erkenntnisgegenstand nicht überwunden. Diese Spaltung verlagert sich dann nur auf Differenzen innerhalb des Diskurses. ${ }^{14}$ Sofern aber die Diskursanalyse keine Lösung des erkenntnistheoretischen Grundproblems bringt, steht sie epistemologisch mit den gegenständlichen Religionsdefinitionen auf ein und derselben Ebene. Dann kann man sich zur Erfassung des Gegenstandes der historischen und sozialwissenschaftlichen Religionsforschung auch dieser bedienen.

Neben diesem erkenntnistheoretischen Einwand muss aber auch eine inhaltliche Kritik vorgebracht werden. Geht es den gegenständlichen Begriffsbestimmungen von Religion darum, zentrale Merkmale dieses Phänomens herauszuarbeiten, so zielt die Analyse des Alltagsverständnisses von Religion auf Diskurse über Religion. Diskurse über Religion und religiöse Vorstellungen, Praktiken oder Zugehörigkeiten sind aber durchaus etwas Verschiedenes, etwa so unterschiedlich wie das Reden über Sex und der sexuelle Akt. Kurz gesagt: Es gibt eben doch eine Wirklichkeit jenseits des Diskurses, auch wenn wir sie diskursiv nicht voll einholen können. Die Gegenstandsbestimmung von Religion zielt auf diese Wirklichkeit, die Diskursanalyse hingegen auf das Re-

\footnotetext{
${ }^{14}$ Das weiß auch Bergunder (2011: 43), der davon ausgeht, ,dass auch die historiographische Konstatierung einer Sedimentierung selbst eine hegemoniale Schließung ist", also ein zwar notwendiger, aber unmöglicher Akt, der die Differenz zwischen Intention und Zielpunkt, erkennendem und zu erkennendem Diskurs verschleiert.
} 
den über Religion. Im diskursanalytischen Ansatz liegt eine Kategorienverwechslung vor.

Schließlich ist das Unternehmen einer genealogischen Diskursanalyse von Religion ähnlich umfassend angelegt wie die Erstellung eines allgemeingültigen Religionsbegriffes, so dass der Vorwurf, es sei ausgeschlossen, das gesamte religionsgeschichtliche Material in seiner Mannigfaltigkeit in der Begriffsbestimmung zu berücksichtigen, auch die Diskursanalyse trifft.

Immerhin ist mit dem diskursanalytischen Ansatz aber doch eines erreicht. Erkenntnistheoretische Naivität ist nach den Einwänden der Diskursanalyse bei jeder zukünftigen Arbeit am Religionsbegriff ausgeschlossen. Wer sich auf dieses Unternehmen einlässt, kann wissen, dass die Differenz zwischen Begriff und Sache prinzipiell unüberbrückbar ist und er sich mit seinem Vorhaben auf das unendliche Spiel der Unterscheidungen eingelassen hat, das nie zu einem befriedigenden Ende kommen kann.

\section{Eine funktional-substanzielle Religionsdefinition}

Eingedenk des diskurstheoretisch formulierten grundsätzlichen epistemologischen Vorbehalts wollen wir uns nun dem Versuch der Erstellung einer eigenen Religionsdefinition zuwenden. Es ist klar, dass diesem Versuch nur ein hypothetischer Charakter zukommen kann. Ob der erstellte Religionsbegriff brauchbar ist, muss sich immer wieder erst in der empirischen Arbeit erweisen. Indem auf diese Weise von Vornherein zwischen theoretischen Überlegungen und empirischer Forschung unterschieden wird, ist dann allerdings auch der Weg frei für eine theoretische Setzung, die trotz der in ihr unausweichlich implizierten alltagsweltlich gewonnenen Beobachtungen die Beurteilung ihrer empirischen Zweckmäßigkeit einem zweiten Schritt vorbehält.

Wenn an dieser Stelle funktionalistische und substanzielle Definitionsmethode miteinander kombiniert werden sollen, ${ }^{15}$ dann

\footnotetext{
${ }^{15}$ Die Verknüpfung funktionaler und substanzieller Argumente wird immer wieder gefordert (so schon Dux 1973: 19-20). Der hier vorgebrachte Vorschlag schließt an Luhmann (1977: 33) an und nimmt Überlegungen von Pollack (1995) auf.
} 
wird damit an die in der Religionssoziologie und der Religionsphilosophie gebräuchliche Dimensionsforschung oder polythetische Definitionsmethode ${ }^{16}$ angeknüpft. Diese verzichtet gleichfalls darauf, den Religionsbegriff durch Rekurs auf ein einziges Merkmal zu bestimmen, und sieht stattdessen eine Vielzahl von Merkmalen als religionskonstitutiv an.

In dem Versuch, eine funktionale Religionsbestimmung mit einem substanziellen Religionsbegriff zu verbinden, muss sowohl das Problem benannt werden, für das Religion Formen der Bearbeitung bereitstellt, als auch die Spezifik der Form erfasst werden, in der sie es bearbeitet. Während mit der Angabe des Bezugsproblems der Religion ein funktionaler Ansatz gewählt wird, nimmt die Analyse der Art und Weise, in der Religion mit dem ausgemachten Problem umgeht, eine substanzielle Begriffsbestimmung vor.

Im Anschluss an Luhmann, Habermas, Lübbe und andere (Luhmann 1977; Habermas 1979: 163 ff.; Lübbe 1986) sei angenommen, dass das Bezugsproblem der Religion im Problem der Kontingenz und Sinnhaftigkeit allen Daseins besteht. Natürlich ließen sich auch andere Probleme ausmachen, auf die religiöse Sinnformen bezogen sind. Der Vorschlag, mit dem Problem der Kontingenz anzusetzen, ist eben nur eine empirisch zu überprüfende Hypothese. Kontingenz meint, dass etwas möglich, aber nicht notwendig ist, dass es ist, was es ist, aber auch ganz anders sein könnte (Aristoteles Met IX 3, 1047a: 20-26). Kontingenz ist also durch die gleichzeitige Negation von Notwendigkeit und Unmöglichkeit definiert. Sozialstrukturell entsteht das Kontingenzproblem durch die Selektivität aller sozialen Strukturen und Prozesse. Soziale Strukturen und Prozesse stehen stets in einem Horizont weiterer Möglichkeiten, die durch sie nicht realisiert sind, aber doch gleichwohl realisiert werden könnten. Sie sind mithin notwendig kontingent, weshalb das Bezugsproblem der Religion an allen Strukturen, Prozessen und Ereignissen aufbrechen kann.

\footnotetext{
${ }^{16}$ Vgl. Glock 1962; Glock/Stark 1968; Trillhaas 1972; Kaufmann 1989; Wilson 1998; Saler 1993.
} 
Von einem funktionalen Bezugsproblem im strengen Sinne ist nur dann zu reden, wenn es im Rücken der Handelnden liegt und von diesen nicht wahrgenommen wird. Kontingenz kann auf der individuellen Ebene jedoch auch perzipiert und erlebt werden. Dann handelt es sich genau genommen nicht mehr um ein funktionales Bezugsproblem, sondern um ein Problem, das zum bewussten Referenzpunkt individuellen Handelns, Wissens und Erlebens geworden ist. Kontingenzprobleme müssen jedoch nicht ins Bewusstsein treten, auch wenn religiöse Praktiken und Überzeugungen und Identitäten als Formen ihrer Bearbeitung interpretiert werden können. Zuweilen ist ihre Latenz sogar eine Voraussetzung für den wirksamen Umgang mit ihnen. Auch auf der Handlungsebene gilt: Kontingenzprobleme können in jeder Situation aufkommen: Warum ist etwas so, wie es ist, warum ist es nicht anders? Warum musste gerade mir dies passieren? Warum gerade jetzt? Warum gerade das? Warum ging diese Liebe zu Ende? Warum wurde ich nicht zum Vorstellungsgespräch eingeladen? Warum habe ich mich im Datum geirrt? Das Problem der Kontingenz, auf das sich das religiöse Erleben und Handeln bezieht, ist von universaler Relevanz.

Die Voraussetzung für seine Wahrnehmung besteht in dem Bewusstsein anderer Möglichkeiten, die im Augenblick zwar nicht aktualisierbar, prinzipiell aber denkbar sind. Das Wissen um die Kontingenz ist damit an die Weite des vorstellbaren Welthorizonts gebunden. Je mehr Möglichkeiten ins Bewusstsein treten, desto höher das Kontingenzbewusstsein.

Der Umgang mit Kontingenz ist jedoch nicht nur ein Bewusstseinsphänomen. Kontingenz ist auch emotional erfahrbar, zum Beispiel wenn etwas Unerwartetes, etwas Gutes oder Schlechtes, geschieht, das das Gemüt des Menschen bewegt. Die affektive Erfahrbarkeit von Kontingenz ist nicht an die Weite, sondern die Begrenztheit des Erwartungshorizonts gebunden. Die Wahrscheinlichkeit von Kontingenzerfahrungen wächst, wenn sich der Mensch in einem geschlossenen Sinnkosmos bewegt, wenn er nicht alles für möglich, sondern manches für ausgeschlossen hält und einem geordneten Weltbild anhängt. Die affektive Kontingenzerfahrung kommt häufig in Gefühlen von Furcht und Hoff- 
nung, von Trauer und Freude, von Bedauern und Dankbarkeit, von Bedrückung und Erleichterung zum Ausdruck.

Die Möglichkeit der Erfahrung von Kontingenz ist stark durch die individuelle Handlungsmacht über das eigene Leben bedingt, von der Fähigkeit, über das eigene Leben selbstmächtig zu bestimmen, Krisen zu bewältigen und Einfluss auf äußere Lebensumstände auszuüben. Wenn Menschen Situationen der Ohnmacht, der Hilflosigkeit und des Unvermögens ausgeliefert sind, wenn sie zum Beispiel konfrontiert sind mit Krankheit, Tod, Armut, unaufhaltsamen sozialem Abstieg, Ungerechtigkeit, mit dem Zerbrechen sozialer Beziehungen oder unerklärlichen inneren Ängsten, dann kommen sie an die Grenzen ihres handlungspraktischen Vermögens und Erfahrungen von Kontingenz werden wahrscheinlich. Kontingenzerfahrungen können aber auch mit der Bewahrung vor Unglück und Verlust, mit der Überwindung von Schwierigkeiten und überraschendem Erfolg, einem hart erkämpften Sieg und unverdientem Glück verbunden sein. Positive Kontingenzerfahrungen können leichter sinnhaft eingeordnet werden als negative und stellen insofern leichter zu bewältigende Herausforderungen dar als negative Kontingenzerfahrungen, obschon auch Siege und Erfolge Verarbeitungsprobleme aufgeben können und oft schon den Keim von tragischer Verstrickung, von Stolz und Hochmut, von Schuld und Versagen, ja sogar von Untergang und Niederlage in sich tragen. Die affektive Erfahrbarkeit von Kontingenz hängt insofern nicht nur von den unterschiedlichen Grenzen des individuellen Erwartungshorizontes ab, sondern auch von dem jeweiligen Handlungsvermögen des Individuums und damit von variierenden Kontextbedingungen. Zieht man die unterschiedlichen Arten der Kontingenzerfahrung in Rechnung, so lassen sich eine kognitive, eine affektive und eine handlungspraktische Dimension der Kontingenzerfahrung unterscheiden.

Typischerweise bringt die Kontingenzerfahrung ein Gefühl der Unsicherheit und Ungewissheit mit sich. Oft weckt sie ein Bedürfnis nach Sicherheit und Ordnung und nicht selten auch nach 
Beruhigung und Trost. ${ }^{17}$ Mit dem Kontingenzproblem ist also das erfasst, was die Markttheoretiker unter der Demand-Side der religiösen Praxis verstehen (Stark/Finke 2000). Es ist klar, dass das Bedürfnis nach Religion, wenn es von Kontingenzerfahrungen abhängt, nicht konstant ist, sondern nach sich wandelnden Umständen jeweils variiert. ${ }^{18}$

Das Kontingenzproblem selbst kann wohl kaum als religiös bezeichnet werden (so auch Stolz 2008: 258), denn Sinnlosigkeit, Leid und Zufälligkeit oder auch Glück und Erfolg stellen Dimensionen der menschlichen Existenz dar, die nicht als solche religiös sind, und es gibt die verschiedensten Formen des Umgangs mit Kontingenzproblemen: Man kann sich Ideologien und Weltdeutungen zurechtlegen, die die erfahrene Kontingenz, vor allem Unglück und Leid, erklären, man kann sich im Falle von Schmerz, Furcht und Trauer in psychotherapeutische Behandlung begeben, sein Verhalten verändern, Gespräche mit guten Freunden führen, sich in harte Arbeit stürzen oder auch seine Erwartungen zurücknehmen. Religiöse Ideen und Praktiken sind nur eine Möglichkeit neben anderen, mit Kontingenzproblemen umzugehen.

Was unterscheidet religiöse Lösungsformen von anderen Antworten auf das Kontingenz- und Sinnproblem? Religion arbeitet mit der Unterscheidung von Immanenz und Transzendenz. Während alles Immanente erreichbar, hinterfragbar, bezweifelbar und kritisierbar ist, bietet das Transzendente aufgrund seiner Unerreichbarkeit Sicherheit und Nicht-Irritierbarkeit. Mit der Einführung der Unterscheidung von Immanenz und Transzendenz schließt Religion die Horizonte der Welt und überführt das Unbestimmbare der Welt in Bestimmbares. Auf diese Weise macht sie

\footnotetext{
${ }^{17}$ Vgl. Riesebrodt (2000: 45): „Der Deutungs- und Sinngebungszwang der menschlichen Gattung zeigt sich besonders deutlich in Krisensituationen, bei Gefahren und Risiken, beim Zusammenbruch sozialer, moralischer und kognitiver Strukturen, wenn Menschen besonders dramatisch mit ihrer eigenen Macht- und Hilflosigkeit konfrontiert werden.“

${ }^{18}$ Dazu ausführlicher: Detlef Pollack: Der historische Wandel des Kontingenzbegriffs als funktionales Bezugsproblem von Religion, in Pollack (2009: 293-303).
} 
Kontingenz hinnehmbar und steigert sie tragbare Unsicherheit (Luhmann 1972: 250-251).

Auch wenn die Sicherheit, die die Unterscheidung zwischen Immanenz und Transzendenz gewährt, auf der Unerreichbarkeit des Transzendenten beruht, kann Kontingenzbewältigung jedoch nur geleistet werden, wenn das Transzendente zugleich zugänglich ist. Anders wäre es nicht erfahrbar und kommunikabel. Die gleichzeitige Anforderung von Unerreichbarkeit und Zugänglichkeit, die die Transzendenz in den religiösen Vollzügen zu erfüllen hat, wirkt paradox. Sie ist aber das typische Merkmal religiöser Sinnformen. Alle Religionen grenzen Orte, Zeiten, Gemeinschaften, Personen, Handlungen, Kommunikationen aus dem Kontinuitätszusammenhang des Alltags aus und versehen das Ausgegrenzte mit besonderen, oft heilig genannten Qualitäten, die sowohl Bestandteil der Immanenz sind als auch auf Transzendentes verweisen. An diesen ausgegrenzten Wirklichkeitsbereichen wird das Transzendente präsent gehalten. Zwischen Immanenz und Transzendenz wird in Religionen also nicht nur unterschieden; vielmehr wird in der Immanenz ein ausgegrenzter Bereich zum Statthalter der Transzendenz: Jesus ist der Christus, der Koran das wortwörtlich geoffenbarte Wort Allahs, die Ikone das Abbild des Erhöhten, das Opfermahl die leibliche Vereinigung mit Gott. Technisch ausgedrückt: Indem in den religiösen Vollzügen zwischen Immanenz und Transzendenz unterschieden und diese Unterscheidung in die Immanenz wieder eingeführt wird, ist die Unzugänglichkeit der Transzendenz ebenso gewährleistet wie ihre Zugänglichkeit. Durch die Form des re-entry (Luhmann 2000: 83-84) vermittelt Religion zwischen Mensch und Gott, zwischen dem Verfügbaren und dem Unverfügbaren, dem Bestimmten und dem Unbestimmten, ohne eines im andern aufgehen zu lassen. Rückt ihr Transzendenzanspruch die religiösen Formen in einen unhinterfragbaren Status, so erlaubt ihnen ihre Verhaftung in der Immanenz, dass sie fassbar werden. Nur wenn beides garantiert ist - ihr Bezug auf Transzendenz und ihre Verfügbarkeit in der Immanenz können die religiösen Formen ihre Funktion der Kontingenzbewältigung erfüllen. Unter Religion verstehen wir also die Einführung der Differenz von Immanenz und Transzendenz in die Im- 
manenz, durch die das Transzendente kommunikativ erreichbar gemacht wird, sowie die gleichzeitige Abhebung des Transzendenten von allem Immanenten, durch die die religiösen Sinnformen der Kontingenz des Immanenten entzogen werden. Auf diese Weise vermag Religion als Interdependenzunterbrechung zu fungieren und erscheint als eine Form der Bewältigung von Kontingenz. ${ }^{19}$

Für die Vermittlung zwischen Immanenz und Transzendenz stehen unterschiedliche religiöse Sinnformen bereit: Rituale, Gebete, Meditationen, Ikonen, Schreine, Altäre, Prozessionen, Predigten, Heilige Schriften usw. ${ }^{20}$ Wenn man versucht, diese religiösen Sinnformen auf der Individualebene zu klassifizieren, lassen sich im Anschluss an Charles Glock und Rodney Stark (1968, vgl. auch Glock 1962) und andere verschiedene Dimensionen unterscheiden. Glock vertrat die Auffassung, dass es in allen Religionen fünf Dimensionen gebe: die ideologische Dimension (der Glaube an bestimmte religiöse Sätze), die ritualistische Dimension, die Dimension der religiösen Erfahrung, die intellektuelle Dimension, die das Wissen um die religiösen Lehrinhalte und Dogmen zum Inhalt hat, sowie die Dimension der Konsequenzen, in der es um die handlungspraktischen Folgen des Glaubens im alltäglichen Leben geht (Glock/Stark 1965: 19ff.). Die an die Arbeiten Glocks anschließende Dimensionsforschung konzentrierte sich vor allem auf drei Fragen, darauf, ob mit den angegebenen Dimensionen auch alle Dimensionen erfasst sind oder sich weitere eruieren lassen, ob die Dimensionen unabhängig voneinander sind oder einige von ihnen zu einer zusammengefasst werden können und welche der Dimensionen gegenüber den anderen dominant ist (vgl. Roof 1979). Glock selbst reduzierte in einer späteren Untersuchung unter Eliminierung der consequential dimension sein Fünf-Dimensionen-Schema auf ein vierdimensionales Modell,

\footnotetext{
${ }^{19}$ Zur Anwendung des hier entworfenen Religionsbegriffs auf die Entwicklung des Abendmahlsverständnisses im Protestantismus siehe Pollack (2008).

${ }^{20}$ Auch Gemeinschaften, Orden, Kirchen können religiösen Charakter haben, sofern und insoweit sie diese Vermittlungsfunktion wahrnehmen.
} 
behauptete für die übriggebliebenen vier Dimensionen eine relative Unabhängigkeit voneinander und maß der Glaubensdimension die höchste Bedeutung bei (Glock/Stark 1968). Andere fanden mehr als fünf Dimensionen heraus (King 1967; Hilty/Morgan/Burns 1984). So fügte etwa Boos-Nünning (1972) den fünf Dimensionen Glocks eine sechste, die Bindung an die Gemeinde, hinzu - eine naheliegende und sinnvolle Ergänzung. Kecskes/Wolf (1996: 55-66) wiederum wiesen einen hohen statistischen Zusammenhang zwischen der religiösen Erfahrung und der Glaubensdimension auf, konnten eine Korrelation dieser beiden Dimensionen mit einer Skala religiösen Wissens dagegen nicht nachweisen.

Wahrscheinlich ist es sinnvoll, im Anschluss an die Arbeiten von Kecskes/Wolf und anderen die Wissensdimension aus der Definition der individuellen Religiosität auszuschließen. Ob jemand viel über Religionen weiß oder nicht, muss mit seiner eigenen Religiosität, mit der Tiefe seiner religiösen Überzeugungen oder der Intensität seiner religiösen Praxis nicht zusammenhängen. ${ }^{21}$ Inwieweit es angebracht ist, die ethische Dimension in die Religiositätsskala aufzunehmen, lässt sich nicht so eindeutig entscheiden. Selbstverständlich kann ethisches Verhalten von Religiosität stark beeinflusst sein. Moralisches Handeln hat aber durchaus auch andere als religiöse Quellen. Seit Pierre Bayle wissen wir, dass es moralisches Handeln auch unabhängig von Religion geben kann und religiöser Eifer häufig amoralische, ja barbarische Folgen hat. Wahrscheinlich sind wir gut beraten, auch diese Dimension aus der Religiositätsskala herauszunehmen. Schließt man die kognitive und die ethische Dimension vom Begriff der individuellen Re-

\footnotetext{
${ }^{21}$ Huber $(2009: 18,22)$ integriert im Anschluss an Glock die intellektuelle Dimension in sein Religiositätsmodell. Seine vielfach zitierte Zentralitätsskala produziert freilich Artefakte und führt im Effekt zu einer Überschätzung des Religiositätsniveaus der Befragten. Dafür nur ein Beispiel: Nach Hubers Zentralitätsindex sind $78 \%$ der Westdeutschen als religiös oder hochreligiös einzuordnen. Derselben Untersuchung zufolge verstehen sich selbst allerdings nur $18 \%$ der Westdeutschen als ,ziemlich“ oder ,sehr religiös“, während sich $40 \%$ als „wenig“ oder „,nicht religiös“ definieren (Müller/Pollack 2009: 415). Der Rest ist unentschieden oder besetzt eine Mittelposition.
} 
ligiosität aus, zieht man mit Kecskes/Wolf die Glaubens- und Erfahrungsdimension zu einer Dimension zusammen und fügt man mit Boos-Nünning die Kirchenbindungsdimension hinzu, so erhält man drei Dimensionen: die Identifikations- oder Zugehörigkeitsdimension, die Handlungs- oder Praxisdimension sowie die Dimension der religiösen Erfahrungen, Überzeugungen und Vorstellungen. Die Identifikationsdimension geht von der Frage aus, wer sich überhaupt zu einer religiösen Gemeinschaft zählt, mit einer Religion oder Konfession identifiziert, in eine religiöse Gemeinschaft eintritt oder aus ihr austritt und sich mit ihr verbunden fühlt. Die Dimension der religiösen Praxis umfasst Riten und kultische Vollzüge und bildet häufig das Rückgrat einer Religion. Diese Dimension lässt sich durch die Frage nach der Kirchgangshäufigkeit, der Gebets- oder Meditationsfrequenz, der Beteiligung am kirchlichen Leben, die Inanspruchnahme von Kasualien wie Taufe, Trauung, Beerdigung und ähnliche Praktiken erfassen. Zur Abbildung der Dimension des religiösen Glaubens und der religiösen Erfahrung kann man nach dem Glauben an Gott, nach der Akzeptanz religiöser Vorstellungen wie Himmel und Hölle, Auferstehung und Wiedergeburt sowie nach Erfahrungen der Nähe von Gott und Engeln fragen. Ebenso lässt sich der Wandel der religiösen Einstellung zum Beispiel durch Konversionserlebnisse analysieren.

All die angegebenen religiösen Inhalte und Formen haben die Aufgabe, Kontakt zur Transzendenz, wie diese auch immer inhaltlich gefüllt sein mag, herzustellen. Gleichzeitig gehören sie jedoch als menschliche Vollzüge zur Immanenz. Wenn im Zuge der religiösen Wirklichkeitsverdopplung die Dissoziierbarkeit des Geeinten aufgehoben wird, besitzen die religiösen Sinnformen Überzeugungskraft. Im re-entry wird also eine präreflexive Einheit zwischen religiösem Symbol und gemeinter Wirklichkeit hergestellt, die der Diskursivität und Explikation entzogen ist.

Eine solche Einheit lässt sich in der Erfahrungsdimension generieren, aber auch in der Dimension des Handelns, des Wissens und der Identität. In der Erfahrungsdimension erlebt der Mensch diese Einheit als ein Gefühl des Überwältigtseins, als etwas, das ihm unverfügbar erscheint und unmanipulierbar auf ihn zukommt. 
In der Handlungsdimension konstituiert sich diese Einheit in sensomotorisch vollzogenen körperlichen Abläufen, die einen habituellen, körperlich verinnerlichten, geradezu automatischen Charakter besitzen. Auch in der kognitiven Dimension kann es Elemente des Unhinterfragbaren geben, mit Evidenz ausgestattete Überzeugungen, die der Reflexion und Diskursivität enthoben sind und unmittelbar einleuchten. Einen solchen unbefragbaren Evidenzcharakter können aber auch Zustände der Zugehörigkeit und Identität annehmen, die allen Zuschreibungen vorauszuliegen scheinen und sich in affektiven Abgrenzungen ebenso auszudrücken vermögen wie in unreflektierter Zuneigung und Sympathie. Der Mensch steht seinem Leben nicht nur in einer exzentrischen Positionalität gegenüber, sondern lebt stets auch von als selbstverständlich unterstellten Hintergrundannahmen, die seinem Leben jene Gewissheit und Sicherheit geben, die er reflexiv nicht zu erzeugen vermag. In der religiösen Gewissheit wird als Einheit behandelt, was sich analytisch trennen lässt, damit aber seinen unbezweifelbaren Gewissheitscharakter verliert. Angesichts der Gleichzeitigkeit von Immanenz und Transzendenz jeder religiösen Sinnform ist das Gewissheitsproblem jedoch stets latent. Es kann aufbrechen vor allem in Situationen der Konkurrenz und Pluralität und scharfe Gestalt annehmen. In Situationen der Herausforderung ist es daher für Religionen notwendig, ihre Formen mit Unüberbietbarkeitsansprüchen auszustatten. Sie können sie als unerreichbar hinstellen, Kommunikationsbarrieren aufrichten, Einschränkungen der Diskursivität vornehmen, Zonen des Geheimnisvollen konstituieren, Autoritäten etablieren, innere Zirkel und, wenn alles nichts hilft, den Ungläubigen exkommunizieren. Nur durch diese Barrieren der Kommunikation lassen sich die religiösen Formen gegen Kritik und Zweifel schützen.

Diese unterschiedlichen Formen der Vermittlung von Transzendenz und Immanenz müssen in der empirischen Untersuchung von Religion analysiert werden. Es ist ein Unterschied, ob die Vermittlung von Immanenz und Transzendenz vorrangig über Formen der Gemeinschaft und Zugehörigkeit zu sozialen Gruppen gewährleistet wird oder ob religiöse Rituale und Praktiken im Vordergrund stehen oder heilige Schriften, theologische Denkfi- 
guren und Predigten oder ob innere Erfahrungen und Vorstellungen als ausschlaggebend angesehen werden. In Abhängigkeit davon, welche Formen im Mittelpunkt stehen, lassen sich unterschiedliche Religionstypen und innerhalb der Religionen unterschiedliche Einstellungs- und Verhaltensmuster ausmachen.

Die Formulierung eines Religionsbegriffes, wie er hier entwickelt wurde, erlaubt es, Quellen religionsinterner Dynamik anzugeben. Sie liegen dem hier entwickelten Ansatz zufolge einmal in der wechselseitigen Bezogenheit von Kontingenzproblem und mit der Unterscheidung von Immanenz und Transzendenz arbeitender Kontingenzbewältigungspraxis, zum anderen in der Dialektik von Verfügbarkeit und Unverfügbarkeit des Transzendenten. Sofern Kontingenzproblematik und Kontingenzbewältigung positiv aufeinander bezogen sind, ist der Grad der Vitalität von religiösen Praktiken und Überzeugungen hoch. Doch was ist, wenn zwar Kontingenzerfahrungen reichhaltig anfallen und damit auch ein hoher Bedarf für religiöse Antworten, religiöse Angebote aber der Kritik und Ablehnung ausgesetzt und nicht angenommen werden? Und was, wenn zwar religiöse Antworten bejaht werden und religiösen Forderungen gehorcht wird, aber das Bedürfnis nach Religion und die Erfahrung von Kontingenz verloren gegangen sind? Im ersten Fall wird man von religiöser Sehnsucht und Suche sprechen können, im zweiten von religiöser Orthodoxie oder Orthopraxie, aber wohl kaum von einer lebendigen und eigendynamischen Religiosität.

Was die Dialektik von Unverfügbarkeit und Verfügbarkeit des Transzendenten angeht, so ergibt sich die religionsinterne Dynamik aus dem Wechselspiel zwischen der Kommunikabilität religiöser Sinnformen und der Unzugänglichkeit des damit Gemeinten. In bestimmten Epochen der Religionsgeschichte dominiert die verdinglichende Symbolisierung des Transzendenten, in anderen wird mehr der Abstand zwischen Gott und Mensch und die uneinfangbare Souveränität des Göttlichen betont. Jedenfalls ist es dem hier skizzierten Ansatz zufolge plausibel, dass zwischen der Konkretisierung des Transzendenten und seiner Verflüssigung ein Spannungsverhältnis besteht und die religionsgeschichtliche Ent- 
wicklung zwischen der Steigerung der Transzendenzspannweite und ihrer Einschränkung hin und her pendelt.

Die hier angedeuteten religionsinternen Dynamiken müssen in der Analyse religiöser Wandlungsprozesse auf religionsexterne Kontextbedingungen bezogen und zu ihnen ins Verhältnis gesetzt werden. Nur so wird es möglich sein, die Faktoren, die den religiösen Wandel beeinflussen, herauszuarbeiten. Zugleich dürfte aber auch deutlich geworden sein, dass es unzureichend ist, allein kontextuelle Umstände zur Erklärung religiöser Wandlungsprozesse heranzuziehen. Die in den religiösen Praktiken, Ideen, Institutionen und Gruppierungen beobachtbaren Spannungsverhältnisse zwischen Kontingenzeröffnung und Kontingenzbewältigung sowie zwischen Immanenz und Transzendenz bedürfen ebenfalls der Berücksichtigung.

\section{Fazit}

Die Stärken der hier vorgeschlagenen Religionsdefinition sind mit ihren Schwächen eng verknüpft. Eine Stärke besteht darin, dass mit ihr Religion als beobachtbares Phänomen von anderen nichtreligiösen Phänomenen abgrenzbar ist. Wo nicht die Unterscheidung von Immanenz und Transzendenz vollzogen wird und diese Unterscheidung in die Immanenz wieder eingeführt wird, handelt es sich nicht um Religion. Überschreitungen der Alltagswelt, Formen der Selbsttranszendenz, philosophische Spekulationen über Gott können folglich noch nicht als religiöse Sinnformen angesprochen werden, da ihnen die Konkretisierung des Transzendenten in der Immanenz, also der re-entry der Unterscheidung von Transzendenz und Immanenz in die Immanenz fehlt. Selbst theologische Ansätze, die allein auf die ungegenständliche Unverfügbarkeit Gottes abstellen, weisen diesem Definitionsansatz zufolge eine Tendenz auf, sich aus dem Kommunikationsraum des Religiösen herauszubewegen. ${ }^{22}$ Der inhaltliche Bestimmung von Religi-

\footnotetext{
${ }^{22}$ Nicht zufällig folgen auf die theologischen Konzepte einer steilen Offenbarungstheologie, die vor allem auf das ganz andere Gottes gegenüber der Welt abheben (Karl Barth), Konzeptionen einer Gott-ist-tot Theologie (vgl. Zahrnt 1968). Diesen Zusammenhang arbeitet Hans Blumenberg (1974) auch schon
} 
on besteht also - um es nochmals zu betonen - nicht nur darin, dass ein von der Immanenz unterschiedener Bereich der Transzendenz eröffnet wird, sondern auch darin, dass dieser Bereich des Unzugänglichen kommunikativ zugänglich, anschaulich, fassbar, verstehbar gemacht wird und damit alltagsweltliche Relevanz erhält.

Zugleich besitzt die vorgeschlagene Religionsdefinition aufgrund der in ihr vorgenommenen Verknüpfung des substanziellen mit dem funktionalen Argument aber auch eine gewisse definitorische Weite. Durch Bezug auf das Kontingenzproblem können religiöse Vorstellungen, Praktiken und Erfahrungen mit nichtreligiösen Kontingenzbewältigungsformen vergleichbar gemacht und die analytischen Perspektiven ausgeweitet werden. Auf der Grundlage dieses Vergleichs kann danach gefragt werden, was religiöse mit anderen Sinnformen gemeinsam haben und sie von ihnen unterscheidet. Die Verbindung eines funktionalistischen und substanziellen Herangehens erlaubt es mithin, die notwendige Bestimmtheit der Religionsdefinition mit einer modalen Weite in der Gegenstandserfassung zu verknüpfen. Die Unterscheidung von Immanenz und Transzendenz und die Symbolisierung der Transzendenz in der Immanenz stellen den unverzichtbaren Kern der Definition dar, der Funktionsbezug eine mögliche, aber nicht notwendige Ergänzung.

Damit wird zugleich deutlich, dass dieser Definitionsvorschlag unmittelbar an das Selbstverständnis der Religionsangehörigen, der Glaubenden und religiöse Praktizierenden anknüpft. Die über Rituale, Gebete, Schriften, Bilder, Tänze, Predigten, Lobgesänge, Erlebnisse oder Erfahrungen ermöglichte Kommunikation mit dem als transzendent Vorgestellten steht für die religiösen Akteure im Zentrum ihrer Religion. Wenn diese religiösen Praktiken, Erfahrungen und Symbole zu dem mit ihnen bearbeiteten Bezugsproblem in Beziehung gesetzt werden, dann wird die Eigenperspektive der Betroffenen freilich überschritten. Der hier vorge-

für die nominalistische Theologie des Mittelalters heraus: Nach der theologischen Übersteigerung der Souveränität Gottes bleibt der Philosophie nur noch die Selbstbehauptung der Vernunft gegen den nominalistischen Gottesbegriff. 
stellte Definitionsvorschlag berücksichtigt das religiöse Selbstverständnis; er übergeht es nicht, macht sich von ihm aber auch nicht abhängig, sondern kann es auch überschreiten. Insofern sind in ihm nicht nur Bestimmtheit und Unbestimmtheit, sondern auch Selbstverständnis und Fremdperspektive zueinander ins Verhältnis gesetzt.

Diese Flexibilität im definitorischen Herangehen impliziert aber auch eine Schwäche. Durch die Aufnahme inhaltlicher und funktionaler Gesichtspunkte ist die Frage danach, welche inhaltlichen Merkmale Religion ausmachen und welche Funktionen sie erfüllen könnte, hypothetisch bereits vorentschieden. In der empirischen Forschung könnten sich jedoch ganz andere Kennzeichen und Funktionsbeziehungen als zentral herausstellen. Die Friedensund Konfliktforschung zum Beispiel könnte herausfinden, dass es Religion weniger mit dem Problem der kognitiven, affektiven oder pragmatischen Kontingenz zu tun hat als mehr mit sozialen und kulturellen Schließungsprozessen oder mit Problemen der Konfliktregulierungen oder mit der Frage nach unverhandelbaren Identitäten. Falls dies oder etwas Ähnliches das Ergebnis empirischer Forschungen sein sollte, könnte sich die Verknüpfung von unterschiedlichen Merkmalen als hinderlich für eine unbefangene Analyse erweisen. Dann würden es die Ergebnisse empirischer Forschung nahelegen, das zur Beobachtung des Gegenstandes entwickelte Kategoriensystem zu verändern. Definitionen sind nicht einfach wahr oder falsch, sondern müssen sich in der empirischen Arbeit als nützlich und zweckmäßig erweisen. Wenn sie einem Gegenstand nicht gerecht werden, müssen sie neu justiert werden. 


\section{Literatur}

Arnal, William Edward 2000: Definition, in: Braun, Willy/McCutcheon, Russel T. (Hrsg.): Guide to the Study of Religion, New York, NY, 21 -34 .

Asad, Talal 1993: Genealogies of Religion. Discipline and Reasons of Power in Christianity and Islam, Baltimore, MD.

Berger, Peter L. 1973: Zur Dialektik von Religion und Gesellschaft. Elemente einer soziologischen Theorie, Frankfurt a. M.

Berger, Peter L. 1974: Some Second Thoughts on Substantive Versus Functional Defintions of Religion, in: Journal for the Scientific Study of Religion 13, 125-133.

Berger, Peter L. 2014: The Many Altars of Modernity, Boston, MA.

Berger, Peter L./Luckmann, Thomas 1966: The Social Construction of Reality. A Treatise in the Sociology of Knowledge, Garden City, NY.

Bergunder, Michael 2011: Was ist Religion? Kulturwissenschaftliche Überlegungen zum Gegenstand der Religionswissenschaft, in: Zeitschrift für Religionswissenschaft 19: 1, S. 3-55.

Blumenberg, Hans 1974: Säkularisierung und Selbstbehauptung. Erweiterte und überarbeitete Neuausgabe von ,Die Legitimität der Neuzeit", erster und zweiter Teil, Frankfurt a. M.

Boos-Nünning, Ursula 1972: Dimensionen der Religiosität. Zur Operationalisierung und Messung religiöser Einstellungen, München.

Bourdieu, Pierre 2009: Religion. Schriften zur Kultursoziologie 5, Konstanz.

Chidester, David 1996: Savage Systems. Colonialism and Comparative Religion in South Africa, Charlottesville, VA.

Dobbelaere, Karel/Lauwers, Jan 1974: Definition of Religion. A Sociological Critique, in: Social Compass 20, 535 - 551.

Dubuisson, Daniel 2003: The Western Construction of Religion. Myths, Knowledge, and Ideology, Baltimore, MD.

Dux, Günther 1973: Ursprung, Funktion und Gehalt der Religion, in: Internationales Jahrbuch für Religionssoziologie 8, 7-67.

Eliade, Mircea 1954: Die Religionen und das Heilige. Elemente der Religionsgeschichte, Salzburg. 
Figl, Johann 2003: Handbuch Religionswissenschaft. Religionen und ihre zentralen Themen, Innsbruck, Tyrolia.

Fitzgerald, Timothy 2000: The Ideology of Religious Studies, Oxford.

Gabriel, Karl 1993: Wandel des Religiösen, in: Forschungsjournal Neue Soziale Bewegungen 6: 3, 24-32.

Glock, Charles Y. 1962: On the Study of Religious Commitment. Religious Education (Research Supplement) 57, 98-110.

Glock, Charles/Stark, Rodney 1965: Religion and Society in Tension, Chicago, IL.

Glock, Charles/Stark, Rodney 1968: American Piety. The Nature of Religious Commitment, Berkeley, CA.

Goody, Jack 1961: Religion and Ritual. The Definitional Problem, in: British Journal of Sociology 12: 2, $142-164$.

Graf, Friedrich W. 2004: Die Wiederkehr der Götter. Religion in der modernen Kultur, München.

Greil, Arthur L. 2009: Defining Religion, in: Clarke, Peter B./Beyer, Peter (Hrsg.): The World's Religions. Continuities and Transformations, Abingdon, 135-149.

Habermas, Jürgen 1979: Legitimationsprobleme im Spätkapitalismus, Frankfurt a. M.

Heiler, Friedrich 1962: Die Religionen der Menschheit, Stuttgart.

Hilty, Dale M./Morgan, Rick L./Burns, Joan E. 1984: King and Hunt Revisited. Dimensions of Religious Involvement, in: Journal for the Scientific Study of Religion 23: 3, $252-266$.

Hock, Klaus 2011: Einführung in die Religionswissenschaft, 4. Auflage, Darmstadt.

Hölscher, Lucian 2005: Geschichte der protestantischen Frömmigkeit in Deutschland, München.

Huber, Stefan 2009: Der Religionsmonitor 2008. Strukturierende Prinzipien, operationale Konstrukte, Auswertungsstrategien, in: Bertelsmann Stiftung (Hrsg.): Woran glaubt die Welt? Analysen und Kommentare zum Religionsmonitor 2008, Gütersloh, 17-52.

Joas, Hans 2004: Braucht der Mensch Religion? Über Erfahrungen der Selbsttranszendenz, Freiburg.

Kaufmann, Franz-Xaver 1989: Religion und Modernität. Sozialwissenschaftliche Perspektiven, Tübingen. 
Kecskes, Robert/Wolf, Christof 1996: Konfession, Religion und soziale Netzwerke. Zur Bedeutung christlicher Religiosität in personalen Beziehungen, Opladen.

King, Morton B. 1967: Measuring the Religious Variable. Nine Proposed Dimensions, in: Journal for the Scientific Study of Religion 6: 2, S. $173-190$.

Kippenberg, Hans G. 1983: Diskursive Religionswissenschaft. Gedanken zu einer Religionswissenschaft, die weder auf einer allgemein gültigen Definition von Religion noch auf einer Überlegenheit von Wissenschaft basiert, in: Gladigow, Burkhard/Kippenberg, Hans G. (Hrsg.): Neue Ansätze in der Religionswissenschaft, München, 9 - 28.

Kippenberg, Hans G./Stuckrad, Kocku von (2003): Einführung in die Religionswissenschaft: Gegenstände und Begriffe, München.

Krech, Volkhard 1998: Religiöse Erfahrung was oder wie? Zur soziologischen Rekonzeptualisierung eines religionswissenschaftlichen Begriffs anhand der Analyse von Konversionsberichten, in: Tyrell, Hartmann/Krech, Volkhard/Knoblauch, Hubert (Hrsg.): Religion als Kommunikation, Würzburg, 473 - 504.

Lambert, Yves 1991: La „Tour de Babel“ des définitions de la religion, in: Social Compass 38, 73 - 85.

Lanczkowski, Günter 1980: Einführung in die Religionswissenschaft, Darmstadt.

Leuba, James H. 1921: The Psychological Origin and the Nature of Religion, London.

Lübbe, Hermann 1986: Religion nach der Aufklärung, Graz.

Luckmann, Thomas 1985: Über die Funktion der Religion, in: Koslowski, Peter (Hrsg.): Die religiöse Dimension der Gesellschaft: Religion und ihre Theorien, Tübingen, $26-41$.

Luckmann, Thomas 1991: Die unsichtbare Religion. Frankfurt a. M.

Luhmann, Niklas 1972: Die Organisierbarkeit von Religionen und Kirchen, in: Wössner, Jakobus (Hrsg.): Religion im Umbruch. Soziologische Beiträge zur Situation von Religion und Kirche in der gegenwärtigen Gesellschaft, Stuttgart, 245 - 285.

Luhmann, Niklas 1977: Funktion der Religion, Frankfurt a. M.

Luhmann, Niklas 2000: Die Religion der Gesellschaft, Frankfurt a. M. 
Marquardt, Odo 1985: Religion und Skepsis: Kommentar zu R. Spaemann und Th. Luckmann, in: Koslowski, Peter (Hrsg.): Die religiöse Dimension der Gesellschaft: Religion und ihre Theorien, Tübingen, $42-47$.

Matthes, Joachim 1967: Religion und Gesellschaft. Einführung in die Religionssoziologie I, Reinbek.

Matthes, Joachim 1992: Auf der Suche nach dem „Religiösen“. Reflexionen zur Theorie und Empirie religionssoziologischer Forschung, in: Sociologia Internationalis 20, 129-142.

Matthes, Joachim 2005: Das Eigene und das Fremde. Gesammelte Aufsätze zu Gesellschaft, Kultur und Religion, Würzburg.

Müller, Olaf/Pollack, Detlef 2007: Kirchlichkeit, Religiosität und Spiritualität. West- und osteuropäische Gesellschaften in Zeiten religiöser Vielfalt, in: Bertelsmann Stiftung (Hrsg.): Woran glaubt die Welt? Analysen und Kommentare zum Religionsmonitor 2008, Gütersloh, 411-430.

McCutcheon, Russell T. 1997: Manufacturing Religion. The Discourse on Sui Generis Religion and the Politics of Nostalgia, Oxford.

Norris, Pippa/Inglehart, Ronald 2004: Sacred and Secular. Religion and Politics Worldwide, Cambridge, MA.

Otto, Rudolf 1917: Das Heilige. Über das Irrationale in der Idee des Göttlichen und sein Verhältnis zum Rationalen, Breslau.

Pettazoni, Raffaele 1960: Der allwissende Gott. Zur Geschichte der Gottesidee, Frankfurt a. M.

Pollack, Detlef 1995: Was ist Religion? Probleme der Definition, in: Zeitschrift für Religionswissenschaft 3: 2, 163-190.

Pollack, Detlef 2008: Die Einheit von Immanenz und Transzendenz in der Krise. Religionssoziologische Anmerkungen zum Wandel des evangelischen Abendmahlsverständnisses, in: Große Kracht, Hermann-Josef/Spieß, Christian (Hrsg.): Christentum und Solidarität: Bestandsaufnahmen zu Sozialethik und Religionssoziologie, Paderborn, 361-388.

Pollack, Detlef 2009: Rückkehr des Religiösen? Studien zum religiösen Wandel in Deutschland und Europa II, Tübingen.

Riesebrodt, Martin 2000: Die Rückkehr der Religionen. Fundamentalismus und der „Kampf der Kulturen“, München. 
Riesebrodt, Martin 2007: Cultus und Heilsversprechen. Eine Theorie der Religionen, München.

Roof, Wade Clark 1979: Concepts and Indicators of Religious Commitment. A Critical Review, in: Wuthnow, Robert (Hrsg.): The Religious Dimension. New Directions in Quantitative Research, New York, NY, $17-45$.

Rüpke, Jörg 2007: Historische Religionswissenschaft: Eine Orientierung, Stuttgart.

Sabbatucci, Dario 1988: Kultur und Religion, in: Cancik, Hubert/Gladigow, Burkhard/Laubscher, Matthias (Hrsg.): Handbuch religionswissenschaftlicher Grundbegriffe I, Stuttgart, 43 - 58.

Saler, Benson 1993: Conceptualizing Religion. Immanent Anthropologists, Transcendent Natives, and Unbounded Categories, Leiden.

Schäfer, Heinrich 2009: Pfingstbewegung. Sozialer Wandel und religiöser Habitus, in: Bertelsmann Stiftung (Hrsg.): Woran glaubt die Welt? Analysen und Kommentare zum Religionsmonitor 2008, Gütersloh, 553-608.

Schütz, Alfred 1971: Über die mannigfaltigen Wirklichkeiten, in: ders.: Gesammelte Aufsätze I, Den Haag, 237-298.

Seiwert, Hubert 1981: „Religiöse Bedeutung“ als wissenschaftliche Kategorie, in: Annual Review for the Social Sciences of Religion 5, 57-99.

Spaemann, Robert 1985: Diskussion über Religion und Funktionalismus, in: Koslowski, Peter (Hrsg.): Die religiöse Dimension der Gesellschaft. Religion und ihre Theorien, Tübingen, 48-60.

Spiro, Melford E. 1966: Religion: Problems of Definition and Explanation, in: Banton, Michael (Hrsg.): Anthropological Approaches to the Study of Religion, London, 85 - 126.

Stark, Rodney/Finke, Roger 2000: Acts of Faith. Explaining the Human Side of Religion. Berkeley, CA.

Stausberg, Michael (Hrsg.) 2009: Contemporary Theories of Religion. A Critical Companion, Abingdon.

Stolz, Jörg 2008: Secularization Theory and Rational Choice Theory. An Integration of Macro- and Micro-Theories of Secularization Using the Example of Switzerland, in: Pollack, Detlef/Olson, Daniel V. A (Hrsg.): The Role of Religion in Modern Societies, London, 249 270 . 
Trillhaas, Wolfgang 1972: Religionsphilosophie, Berlin.

Tylor, Edward Burnett 1871: Primitive Culture. Researches into the Development of Mythology, Philosophy, Religion, Art, and Custom, London.

Tyrell, Hartmann 1996: Religionssoziologie, in: Geschichte und Gesellschaft 22: 3, $428-457$.

Waardenburg, Jacques 1984: Über die Religion der Religionswissenschaft, in: Neue Zeitschrift für Systematische Theologie und Religionsphilosophie 26, $238-255$.

Waardenburg, Jacques 1993: Perspektiven der Religionswissenschaft, Würzburg.

Wagner, Falk 1986: Was ist Religion? Studien zu ihrem Begriff und Thema in Geschichte und Gegenwart, Gütersloh.

Widengren, Geo 1969: Religionsphänomenologie, Berlin.

Wilson, Brian C. 1998: From the Lexical to the Polythetic, in: Idinopulos, Thomas A./Wilson, Brian C. (Hrsg.): What is Religion? Origins, Definitions and Explanations, Leiden, 141-162.

Wuthnow, Robert 1988: Sociology of Religion, in: Smelser, Neil J. (Hrsg.): Handbook of Sociology, Beverly Hills, CA, 473 - 509.

Zahrnt, Heinz 1968: Die Sache mit Gott. Die protestantische Theologie im 20. Jahrhundert, München.

Zinser, Hartmut 2010: Grundfragen der Religionswissenschaft, Paderborn. 